\title{
Celiac Disease a Road Paved with Many Obstacles. Differential Diagnosis in Children
}

\author{
Sur M. Lucia ${ }^{1,3}$, Colceriu Marius ${ }^{1}$, Cornel Aldea ${ }^{2, *}$, Sur Genel ${ }^{1,2}$, Floca Emanuela ${ }^{1,4}$ \\ ${ }^{1}$ University of Medicine and Pharmacy “Iuliu Hațieganu” Cluj-Napoca Romania \\ ${ }^{2}$ Emergency Clinical Hospital for Children, Cluj-Napoca - Pediatrics II Department \\ ${ }^{3}$ Emergency Clinical Hospital for Children, Cluj-Napoca - Pediatrics I Department \\ ${ }^{4}$ Regional Institute of Gastroenterology and Hepatology “Octavian Fodor” Cluj-Napoca \\ *Corresponding author: cornelaldea65@yahoo.com
}

\begin{abstract}
Celiac disease (CD), also known as gluten-sensitive enteropathy or celiac sprue, is defined as a chronic, multifactorial, immune-mediated condition affecting small intestine in genetically susceptible children and adults. Gluten-sensitive enteropathy is characterized by heterogeneous clinical manifestations of variable severity that can occur at any age. For patients with prompt diagnosis and correct treatment the prognosis is excellent. Therefore, $\mathrm{CD}$ needs a quick diagnosis in order to start the treatment. Because of the wide range of signs and symptoms, an accurate differential diagnosis is required.
\end{abstract}

Keywords: celiac disease, differential diagnosis, classical, non-classical, silent (asymptomatic)

Cite This Article: Sur M. Lucia, Colceriu Marius, Cornel Aldea, Sur Genel, and Floca Emanuela, "Celiac Disease a Road Paved with Many Obstacles. Differential Diagnosis in Children.” International Journal of Celiac Disease, vol. 6, no. 1 (2018): 7-10. doi: 10.12691/ijcd-6-1-5.

\section{Introduction}

Celiac disease (CD), also known as gluten-sensitive enteropathy or celiac sprue, is defined as a chronic, multifactorial, immune-mediated condition affecting small intestine in genetically susceptible children and adults. Manifested as an enteropathy, it is triggered by gluten ingestion, which is a protein founded in grains as wheat, rye or barley. For these individuals, exposure to gluten or similar proteins (secalins, gliadins, hordeins) induces mucosal impairments represented by intraepithelial lymphocytosis, crypt hyperplasia and villous atrophy. Gluten-sensitive enteropathy is characterized by heterogeneous clinical manifestations of variable severity that can occur at any age. $[1,2,3]$

\subsection{Epidemiology}

The worldwide prevalance of CD is not uniform between different countries. A greater prevalence has been found in developed countries ( $1 \%$ in general population). For example, one study made in Sweden showed a prevalence of $2 \%$ in general population. In children, CD appears most frequently between 9-18 months of life. Some studies made in United States and Europe showed a prevalence in children population around 3-13/1000 children. [4,5,6]

\subsection{Presentation}

Clinical manifestation of $\mathrm{CD}$ in pediatric patients is characterized by a wide range of signs and symptoms. According to presented simptomatology, CD can be divided in classical subtype with gastrointestinal manifestations and non-classical subtype with extraintestinal manifestations. There are also 3 more subtypes of known CD: silent CD defined by mucosal impairments, positive serology, but without symptoms; potential CD defined by positive serology, normal intestinal mucosa, with or without symptoms; latent $\mathrm{CD}$ in which the patient is without symptoms at the time of presentation and the mucosal morphology is normal, but they have had CD at some point in the past $[7,8]$.

\subsection{Diagnosis}

Patients with high-risk or symptoms need to be tested for anti-transglutaminase autoantibodies (IgA TTG). To confirm the diagnosis, for patients with positive serology, biopsies are needed. If the patient is under 2 years, IgG-deamidated gliadin peptides test should be added to serological testing. Another important criterion in CD diagnosis is represented by remission under gluten-free diet (GFD) and relapses after reintroduction of gluten $[9,10]$.

\subsection{Treatment}

Lifelong strict removal of gluten from diet represents the mainstay of treatment. A GFD leads to symptom resolution, normalization of mucosal morphological aspect and serological markers. New therapeutic perspectives are developed in order to improve prognosis of refractory CD. $[11,12]$. 


\subsection{Prognosis}

For patients with prompt diagnosis and correct treatment the prognosis is excellent. Morbidity and mortality are higher in patients with CD compared to general population especially if the diagnosis is delayed or the patients do not have a GFD. If this condition is not treated, it can lead to failure to thrive, osteoporosis, infertility, delayed puberty, or even adenocarcinomas or lymphomas. $[13,14]$

As shown above, CD is a relative frequent pathological condition that needs a quick diagnosis in order to start the treatment. Because of the wide range of signs and symptoms, an accurate differential diagnosis is required $[15,16]$. So our goal was to make a current analysis of the differential diagnosis of CD.

\section{Differential Diagnosis}

\subsection{Classical Subtype of CD}

Classical subtype of CD is characterized by gastrointestinal signs and symptoms such as chronic diarrhea, abdominal pain and distension, vomiting, anorexia, muscle wasting, weight loss, failure to thrive. The onset of symptoms typically occurs between 9 and 24 months of age, after introduction of gluten in diet. Older children may have onset of manifestations at any age. If $\mathrm{CD}$ is not treated severe malnutrition and celiac crisis (explosive diarrhea, abdominal distension, electrolyte imbalance, dehydration, hypotension, lethargy) can occur. On physical examination patients are often pale, underweight, with proeminent abdomen. They also may present edemas due to hypoalbuminemia and flattened buttocks. [17]. The differential diagnosis for this subtype of CD includes autoimmune enteropathy, cystic fibrosis, inflammatory bowel disease (Crohn disease), pediatric irritable bowel syndrome, protein intolerance, giardiasis, eosinophilic gastroenteritis, bacterial or viral gastroenteritis. [18]

\subsubsection{Autoimmune Enteropathy (AE)}

Autoimmune enteropathy (AE) is a rare pathological condition, typically occuring in first weeks of life, defined by severe diarrhea that leads to malabsorption and growth retardation. In the pathogenesis of this disease, it has been incriminated an immunological mechanism caused by disfunction of regulatory T-cells that determine production of antienterocyte autoantibodies. The histopathological examination of intestinal mucosa shows some analogies with CD: villous atrophy, crypt hyperplasia and lymphoplasmacytic infiltrate. Unlike CD, there is a paucity of intraepithelial lymphocytes and the impairments of mucosa can be extended to the whole gastrointestinal tract. As in CD, there can occur extraintestinal manifestation such as diabetes mellitus, liver impairments, arthritis or cutaneous manifestation (in this case severe allergies and eczema). The diagnostic work-up of AE includes clinical presentation, serological tests and histopathological examination. Because of the similarities regarding clinical aspects, histopathological pattern and association with autoimmune diseases, between $C D$ and $A E$, a correct differential diagnosis is mandatory. Thus, a GFD test is needed. $[19,20]$

\subsubsection{Cystic Fibrosis (CF)}

Cystic fibrosis (CF) is a chronic and progressive disease in which exocrine glands function is alterated, so they produce abnormally thick, sticky mucus. This disfunction leads to digestive, respiratory and body cooling impairments. CF is a genetic disease caused by mutations in CFTR gene, which determine an abnormal regulation of chloride secretion and sodium absorption. Around $70 \%$ of patients are diagnosed in the first year of life. CF presents gastrointestinal manifestations: meconium ileus, steatorrhea, malabsorption, flatulence, abdominal pain and distention, jaundice, failure to thrive, rectal prolapse. Also, respiratory signs and symptoms are present: chronic cough, bronchiolitis, recurrent wheezing, pneumonia, pneumothorax, atypical asthma, hemoptysis, chest pain, sinusitis, digital clubbing, nasal polyps. In males can occur sterility, hydrocele or undescended testicles. CF diagnosis is based on family history, typical gastrointestinal and respiratory manifestations and a positive sweat chloride test or a positive genetic test. In the past $\mathrm{CF}$ and $\mathrm{CD}$ were considered a single clinical entity, because malabsorption syndrome occurs in both and clinical presentation may be similar. More than that, in malabsorption syndrome the sweat test may be false positive, which makes the differential diagnosis more difficult. This is why a complete and accurate diagnosis is needed. [21,22]

\subsubsection{Crohn Disease}

Crohn disease is a inflammatory bowel disease with chronic evolution that may affect any part of the gastrointestinal tract. There are some overlapping symptoms between CD and Crohn disease such as diarrhea, abdominal pain, weight loss, growth deceleration, anorexia, arthritis, pubertal delay, bone demineralization. On the other hand, there are some manifestations that may occur just in Crohn disease: episcleritis, anterior uveitis, erythema nodosum, pyoderma gangrenosum, perianal disease. Endoscopy aspect of Crohn disease and biopsy are helpful in differentiating between CD and Crohn disease. Histological examination reveals transmural inflammation, architectural crypt changes, cryptitis and granulomas. Sometimes distinguishing $\mathrm{CD}$ and Crohn disease based just on symptoms could be quite difficult. In these cases the endoscopic examination with biopsy, serological screening (ASCA) and GFD will establish the diagnosis. [23,24]

\subsubsection{Pediatric Irritable Bowel Syndrome}

Pediatric irritable bowel syndrome is another pathological entity that needs to be distinguished from CD, which is characterized by recurrent abdominal pain, diarrhea or constipation, bloating, without an organic abnormality to explain these manifestations. This disease is a functional disorder with no identifiable cause. Abdominal pain in this disorder is usually located in the periumbilical or hypogastric regions, has no specific pattern and can be exacerbated by stress or food, and partially relieved after defecation. Also, extraintestinal symptoms can be present, such as dysmenorrhea, incomplete bladder emptying, urinary frequency, back pain or headache. But these complaints are rare in children. The diagnosis includes the presence of characteristic symptoms and exclusion of other pathological entities with similar clinical manifestations. [25,26] 


\subsubsection{Protein Intolerance}

Protein intolerance represents alloffending reactions to food proteins, regardless the pathogenesis. The most frequent proteins implicated are cow's milk proteins (during infancy), soybean proteins (infants fed with soy formula) and egg proteins (school age). Clinical presentation includes gastrointestinal symptoms which are the most common, cutaneous symptoms and respiratory symptoms. Typically patients present vomiting and diarrhea. In some cases they can become dehydrated and lose weight. In case of developed enteropathy, a malabsorption syndrom occurs with growth failure. The diagnosis is based on atopic familiar history, symptoms, serum specific IgE, skin prick test with food extract, fecal leukocyte testing, elimination diets, food challenge testing. $[27,28]$

\subsubsection{Giardiasis}

Giardiasis is a diarrheal condition caused by an intestinal parasite, Giardia intestinalis. This parasite can cause asymptomatic disease or diarrheal illness (acute or chronic). In addition, this condition can lead to growth retardation. Other symptoms could be weakness, flatulence, abdominal distention and cramps, nausea, anorexia, weight loss and urticaria. The classical mode to diagnose giardiasis is the identification of parasite or cysts in the stool. Stool antigen enzyme-linked immunosorbent assay (ELISA) and stool culture are also available. [29,30]

\subsubsection{Eosinophilic Gastroenteritis}

Eosinophilic gastroenteritis is a rare condition defined by eosinophilic infiltration of the intestinal wall, especially at stomach an duodenum levels. Clinical manifestations are abdominal pain and cramps, growth retardation, diarrhea, vomiting, failure to thrive, malabsorption syndrome. Diagnosis requires presence of symptoms, eosinophilic infiltration on biopsy specimens and the absence of a known cause of eosinophilia. [31,32]

\subsubsection{Bacterial or Viral Gastroenteritis}

Bacterial or viral gastroenteritis are infectious diseases with high frequency in pediatric population. Because of its clinical manifestations infectious gastroenteritis has to be distinguished from CD. Clinical spectrum ranges from asymptomatic to severe dehydration or death. Usually the symptoms are vomiting, diarrhea and abdominal pain and cramps. In bacterial infections, high fever, severe abdominal pain and bloody diarrhea may occur. Generally if a viral infection is suspected, laboratory tests are not indicated. In other cases stool pH, fecal leukocytes, stool culture, microscopy examination could be useful. $[33,34]$

\subsection{Non-classical Subtype of CD}

Non-classical subtype of CD occurs in more than half of cases and is characterized by extraintestinal signs and symptoms such as dermatitis herpetiformis, dental enamel hypoplasia, osteopenia/osteoporosis, delayed puberty, Iron-deficiency anemia resistant to oral Fe, arthritis, chronic hepatitis and hypertransaminasemia, neurologic
problems.Just like in classical CD, this subtype of CD has to be distinguished especially from Crohn disease because these disorders share a wide range of similar extraintestinal manifestations (anemia, arthritis, osteopenia/osteoporosis, cutaneous manifestations). [17]

\subsubsection{Dermatitis Herpetiformis}

Dermatitis herpetiformis is a blistering skin disease characterized by an erythematous, pruriticeruption, grouped excoriations, urticarial plaques, papules and vesicles. Usually the eruption is localized on elbows, knees, back and buttocks. The eruption slowly disappears after a GFD. Differential diagnosis for dermatitis herpetiformis should include Bullous Pemphigoid, Erythema Multiforme, Excoriation Disorder, Linear IgA Dermatosis, Papular Urticaria, Scabies, Transient Acantholytic Dermatosis. $[35,36]$

\subsubsection{Manifestation}

When CD manifestation are dominated by arthritis and arthralgia, a differential diagnosis should be made with juvenile rheumatoid arthritis. This condition describes a group of arthritides of unknown cause lasting more than 6 weeks. Additional manifestations may be spiking fevers, dermatologic manifestations (rash, psoriasis). Juvenile rheumatoid arthritis is a diagnosis of exclusion based on family and personal history and physical examination. Also, inflammatory markers, complete blood count, liver function tests, antinuclear antibody testing, rheumatoid factor or anti-cyclic citrullinated peptide antibody could be useful. [37,38]

\subsection{Silent (Asymptomatic) Form of CD}

Silent (asymptomatic) form of CD is defined by the presence of histologic changes, in children apparently asymptomatic. These cases have been identified after an endoscopy for another pathologic condition or through screening programs. In this situation a differential diagnosis with villous atrophy should be made. Villous atrophy can occur in tropical sprue, hypogammaglobulinemia, idiopathic AIDS enteropathy, food protein hypersensitivity, eosinophilic gastroenteritis, Whipple disease, intestinal lymphoma, collagenous sprue, tuberculosis, giardiasis, Crohn's disease, small-bowel bacterial overgrowth, infectious enteritis, parasitic infestation, severe malnutrition. [17]

\section{Conclusions}

CD is not difficult to diagnose when it manifests classic symptoms, but in practice, in $70 \%$ of cases, it occurs with atypical symptoms which makes the diagnosis much more difficult. This is why an accurate and complete differential diagnosis is required. The differential diagnostic evaluation should be made with targeted laboratory tests, endoscopy, biopsy and imaging tests if necessary, rationally based on the nutritional history, the patient's weight graph, and the clinical manifestations. It represents a key process to an accurate and complete diagnosis of CD, which is the first step for the right treatment. 


\section{References}

[1] Scanlon SA, Murray JA. Update on celiac disease - etiology, differential diagnosis, drug targets, and management advances. Clinical and Experimental Gastroenterol 2011; 4: 297-311.

[2] Samasca G, Sur G, Lupan I. Current Trends and Investigative Developments in Celiac Disease. Immunological Investigations 2013; 42(4), 273-284.

[3] Polanco I. Celiac Disease in Children. In Rodrigo L and Peña AS, editors. Celiac Disease and NonCeliac Gluten Sensitvity. Barcelona, Spain: OmniaScience; 2014. p. 221-233.

[4] Lionetti, E., Castellaneta, S., Francavilla, R., Pulvirenti, A., Tonutti, E., Amarri, S., Barbato, M., Barbera, C., Barera, G., Bellantoni, A., Castellano, E., Guariso, G., Limongelli, M., Pellegrino, S., Polloni, C., Ughi, C., Zuin, G., Fasano, A. and Catassi, C. Introduction of Gluten, HLA Status, and the Risk of Celiac Disease in Children. New England Journal of Medicine 2014; 371(14): 1295-1303.

[5] Samasca G, Pirvan A, Sur G, Abrudan C, Lupan I, Deleanu D. IgA Tissue Transglutaminase Antibodies at Different cut-offs in the Evaluation of Possible Celiac Patients. International Journal of Celiac Disease 2016; 4(2): 61-63.

[6] Emedicine.medscape.com. (2018). Pediatric Celiac Disease: Background, Pathophysiology, Epidemiology. [online] Available at:

https://emedicine.medscape.com/article/932104-overview [Accessed 12 Jan. 2018].

[7] Sur G, Floca E, Sur L, Sur D, Samasca G. Clinical Presentation of Celiac Disease Masks Therapeutic Perspectives of Celiac Disease. Pharmaceutica Analytica Acta. 2013; 04(04): 1000228.

[8] Hahn M, Hagel A, Hirschmann S, Bechthold C, Konturek, P, Neurath M, Raithel M. Modern diagnosis of celiac disease and relevant differential diagnoses in the case of cereal intolerance. Allergo Journal International 2014; 23(2): 67-77.

[9] Rubio-Tapia A, Hill I, Kelly C, Calderwood A, Murray, J. ACG Clinical Guidelines: Diagnosis and Management of Celiac Disease. American Journal of Gastroenterology 2013; 108(5): 656-676.

[10] Samasca G, Sur G, Man S, Lupan I, Deleanu D. Are the Current Biomarkers Sufficient for Celiac Disease Diagnosis? Medical Connections/Conexiuni Medicale 2014; 9(4): 47-51.

[11] Samasca G, Lupan I, Deleanu D, Cristea V, Makovicky P. Immunological approach of the challenges of the XXI century in celiac disease. International Reviews of Immunology 2014; 33(1): 3-8.

[12] Lebwohl B, Sanders D, Green P. Coeliac disease. The Lancet 2018; 391(10115): 70-81.

[13] Samasca G, Lerner A, Girbovan AM, Sur G, Lupan I, Makovicky P, Matthias T, H.J. Freeman. Challenges in Gluten-free diet in celiac disease: Prague consensus. European Journal Of Clinical Investigation 2017: 47(5): 394-397.

[14] Emedicine.medscape.com. (2018). Celiac Disease (Sprue): Practice Essentials, Background, Pathophysiology. [online] Available at: https: //emedicine.medscape.com/article/171805overview\#a3 [Accessed 13 Jan. 2018]

[15] Samasca G, Sur G, Lupan I, Tilinca M, Deleanu D. Celiac disease as an autoimmune condition. Central-European Journal of Immunology 2014; 39(3): 396-399.

[16] Sur G, Pirvan A, Deleanu D, Samasca G, Lupan I. News in Celiac Disease about Differential Diagnosis from Romania. International Journal of Celiac Disease 2014; 2(2): 40-43.

[17] Fasano A, Catassi C. Current approaches to diagnosis and treatment of celiac disease: An evolving spectrum. Gastroenterology 2001; 120(3): 636-651.

[18] Schuppan D, Zimmer K-P. The Diagnosis and Treatment of Celiac Disease. Deutsches Ärzteblatt International. 2013; 110(49): 835-846.
[19] Montalto M, D'Onofrio F, Santoro L, Gallo A, Gasbarrini A, Gasbarrini G. Autoimmune enteropathy in children and adults. Scandinavian Journal of Gastroenterology 2009; 44(9): 1029-1036.

[20] Lachaux A, Loras-Duclaux I,.Bouvier R. Autoimmune enteropathy in Infants. Virchows Archiv 1998: 433(5): 481-485.

[21] Ramos A, Figueirêdo M, Aguiar A, Almeida C, Mendes P, Souza E. Celiac Disease and Cystic Fibrosis: Challenges to Differential Diagnosis. Folia Medica 2016: 58(2).

[22] Emedicine.medscape.com. (2018). Cystic Fibrosis: Practice Essentials, Background, Pathophysiology. [online] Available at: https://emedicine.medscape.com/article/1001602-overview\#a1 [Accessed 14 Jan. 2018].

[23] Pascual V. Inflammatory bowel disease and celiac disease: Overlaps and differences. World Journal of Gastroenterology 2014 20(17): 4846

[24] Emedicine.medscape.com. (2018). Pediatric Crohn Disease: Practice Essentials, Background, Pathophysiology. [online] Available at: https: //emedicine.medscape.com/article/928288overview [Accessed 14 Jan. 2018].

[25] Girbovan A, Sur G, Samasca G, Lupan I. Dysbiosis a risk factor for celiac disease. Medical Microbiology And Immunology. 2017; 206(2): 83-91.

[26] Emedicine.medscape.com. (2018). Pediatric Irritable Bowel Syndrome Clinical Presentation: History, Physical, Causes. [online] Available at: https: //emedicine.medscape.com/article/930844clinical [Accessed 14 Jan. 2018].

[27] Samasca G, Sur G, Iancu M, Lupan I, Deleanu D. Prevalence of specific IgE to wheat flour allergens in romanian pediatric population. Iranian Journal of allergy, asthma, and immunology 2015; 14(5): 543-544.

[28] Samasca G, Sur G, Iancu M, Lupan I, Deleanu D. Current trends and investivative developments in wheat allergy. International Reviews of Immunology 2015; 34(6): 538-541.

[29] Giardiasis Workup: Approach Considerations, Stool Examination, Stool Antigen Detection [Internet]. Emedicine.medscape.com. 2018 [cited 14 January 2018]. Available from: https://emedicine.medscape.com/article/176718-workup

[30] Girbovan AM, Samasca G, Lupan I. Are Parasitic Infections a Solution in the Treatment of Celiac Disease? International Journal of Celiac Disease 2017; 5(3): 125-126.

[31] Ingle S. Eosinophilic gastroenteritis: An unusual type of gastroenteritis. World Journal of Gastroenterology. 2013; 19(31): 5061.

[32] Bordea MA, Mosteanu O, Pop TA, Gheban D, Samasca G, Miu N. Eosinophilic esophagitis. Acta Gastro-Enterologica Belgica 2013; 76(Fasc.4): 407-412,

[33] Elliott E. Acute gastroenteritis in children. BMJ. 2007; 334(7583): 35-40.

[34] Diaconescu I, Alexandru G, Carpa R, Lupan I, Crisan C, Sur L, Sur G, Girbovan A. Too Few Studies Provided a Link between Viral Infections and Celiac Disease International Journal of Celiac Disease 2016: 4(4): 135-137.

[35] Dermatitis Herpetiformis Differential Diagnoses [Internet]. Emedicine.medscape.com. 2018 [cited 15 January 2018]. Available from: https: //emedicine.medscape.com/article/1062640differential

[36] Samasca G, Baican A, Pirvan A, Miu N, Dejica D. Celiac Disease with Dermatitis Herpetiformis Case Report. Journal of Biomolecular Research and Therapeutics 2012; 1(1): 1000e101.

[37] Spârchez M, Lupan I, Delean D, Bizo A, Damian L, Muntean L, Tămas MM, Bolba C, Simionescu B, Slăvescu C, Felea I, Lazăr C, Spârchez Z, Rednic S. Primary complement and antibody deficiencies in autoimmune rheumatologic diseases with juvenile onset: A prospective study at two centers. Pediatric Rheumatology 2015; 13: 51.

[38] Giancane G, Consolaro A, Lanni S, Davì S, Schiappapietra B, Ravelli A. Juvenile Idiopathic Arthritis: Diagnosis and Treatment. Rheumatology and Therapy. 2016; 3(2): 187-207. 